\title{
Multidisciplinary management of pleural infection after ventricular assist device implantation
}

\author{
Ali Akil ${ }^{1}$, Stephanie Rehers ${ }^{2}$, Christian Köhler ${ }^{1}$, Lars Richter ${ }^{1}$, Michael Semik ${ }^{1}$, Stephan Ziegeler ${ }^{2}$, \\ Stefan Fischer ${ }^{1}$ \\ ${ }^{1}$ Department of Thoracic Surgery and Lung Support, Ibbenbueren General Hospital, Ibbenbueren, Germany; ${ }^{2}$ Department of Anesthesiology, \\ Intensive Care Medicine and Pain Management, Ibbenbueren General Hospital, Ibbenbueren, Germany \\ Contributions: (I) Conception and design: A Akil, S Rehers, S Fischer; (II) Administrative support: S Ziegeler, S Fischer, M Semik; (III) Provision \\ of study materials or patients: A Akil, S Ziegeler, S Rehers; (IV) Collection and assembly of data: A Akil, C Köhler, L Richter, S Rehers; (V) Data \\ analysis and interpretation: A Akil, C Köhler; (VI) Manuscript writing: All authors; (VII) Final approval of manuscript: All authors. \\ Correspondence to: Stefan Fischer, MD, MSc, PhD. Head, Department of Thoracic Surgery and Lung Support, Klinikum Ibbenbueren, Grosse Strasse \\ 41, 49477 Ibbenbueren, Germany. Email: s.fischer@klinikum-ibbenbueren.de.
}

Background: Postsurgical pleural infection is a life-threatening complication after implantation of artificial devices such as ventricular assist devices (VADs). The treatment can be challenging and the evidence in the literature is very limited. Here we report our multidisciplinary approach of the management of pleural infection after VAD implantation.

Methods: Between March 2014 and December 2019, 33 patients developed postoperative pleural infection after VAD implantation and underwent thoracic surgical intervention at our institution. All patients were prospectively enrolled in this analysis. Data were retrospectively analyzed. Primary outcome was the 90-day mortality rate. Length of ICU stay related to pleural infection, chest tube duration, re-thoracotomy rate and length of ventilatory support represented secondary outcomes.

Results: The 90-day mortality rate was 6\% (2 patients). The mean ICU stay related to the pleural infection was 6 days (2-24 days). Video-assisted thoracoscopic surgery (VATS) was performed in all patients. Conversion to thoracotomy was necessary in 12 cases. Decortication and parietal pleurectomy in addition to hematoma and empyema removal was performed in all patients. Due to diffuse bleeding, packing of the thoracic cavity with temporary thoracic closure was necessary in 10 patients. Depacking was performed after a mean of 3 days (3-7 days). Recurrent empyema or bleeding after definitive chest closure was not observed. Lung resection was performed in 3 patients.

Conclusions: Thoracic surgical management of pleural infection in patients after VAD implantation is challenging and complicated due to the inevitable anticoagulative therapy. A perioperative multidisciplinary management which includes the early involvement of thoracic surgical expertise helps to improve survival in this very complex patient cohort.

Keywords: Ventricular assist device (VAD); pleural infection; thoracic surgery

Submitted Sep 14, 2020. Accepted for publication Feb 21, 2021.

doi: $10.21037 /$ jtd-20-2886

View this article at: https://dx.doi.org/10.21037/jtd-20-2886

\section{Introduction}

Mechanical circulatory support (MCS) by ventricular assist devices (VADs) represents a widely accepted and successful treatment option for patients with advanced heart failure (1). The ongoing development of MCS systems from the first fully implantable artificial heart system to modern ventricular support devices, focused on aspects such as pump size, biocompatibility, durability, effectiveness and susceptibility to infection in order to minimize mortality 
Table 1 Baseline characteristics

\begin{tabular}{lc}
\hline Characteristics & VAD patients $(\mathrm{n}=33)$ \\
\hline Age (IQR), years & $65[49-78]$ \\
Gender (male/female) & $31 / 2$ \\
Ethiology of heart failure (\%) & \\
Ischemic cardiomyopathy & $24(72.7)$ \\
Dilated cardiomyopathy & $7(21.2)$ \\
Right ventricular failure & $2(6.1)$ \\
Hypertension (\%) & $28(84.8)$ \\
Diabetes mellitus (\%) & $22(66.6)$ \\
COPD (\%) & $11(33.3)$ \\
Tracheotomy (\%) & $4(12.1)$ \\
LVAD (\%) & $31(93.8)$ \\
Abbott HeartMate 3 & $5(15.1)$ \\
Medtronic HVAD & $26(78.7)$ \\
RVAD (\%) & $2(6.2)$ \\
\hline
\end{tabular}

and adverse events (2). According to a recent INTERMACS report, overall survival after VAD implantation was up to $70 \%$ at 2 years. Bleeding was addressed as major adverse event mostly due to surgical bleeding during the early postoperative phase and gastrointestinal bleeding 3 months after VAD implantation (3). However serious complications of permanent MCS are infections, bleeding, pump thrombosis and cerebrovascular events after VAD implantation (2-5).

The management of postoperative complications in patients with VAD is challenging due to the need for effective anticoagulation, especially when postoperative surgical bleeding with the need for redo thoracotomy or systemic severe inflammation due to infections of the thoracic cavity occur. Among those complications, pleural empyema i.e., due to an infected postoperative hemothorax represent a rare but serious complication. This poses a high risk for primary or secondary device infection through the blood stream if therapy is delayed. Therefore, a multidisciplinary approach which involves the expertise of thoracic surgery and the implanting cardiac surgery center at an early disease state, may help to reduce mortality and to avoid further complications.

Here we present our approach to the thoracic surgical management of postoperative pleural infection in patients after VAD implantation. We present the following article in accordance with the STROBE reporting checklist (available at https://dx.doi.org/10.21037/jtd-20-2886).

\section{Methods}

Between March 2014 and December 2019, 33 patients developed pleural infection, which required thoracic surgical intervention after VAD implantation. In all patients, chest tube placement or thoracentesis had already been performed at various cardiac surgical departments. If the pleural effusion reoccurred or hematoma was not evacuated completely, patients were transferred to the thoracic surgical intensive care unit. The diagnosis of pleural infection was confirmed if a $\mathrm{pH}$ level less than 7.2 and lactate dehydrogenase greater than 1,000 IU/L was determined by pleural fluid analysis (6).

Patients' demographic data, preoperative characteristics and associated comorbidities are summarized in Table 1.

The primary endpoint of this retrospective observation was the 90-day mortality rate. Secondary end points included length of ICU stay related to pleural infection, chest tube duration, re-thoracotomy rate, length of ventilatory support and decannulation after preoperative tracheostomy. All data were collected prospectively and retrospectively analyzed. Continuous variables were described using means, standard deviations, and ranges.

The implanting institutional anticoagulation regimen for patients after VAD implantation was implemented perioperatively. Unfractionated heparin was continuously administered intravenously with a target activated partial thromboplastin time (aPTT) of 50-60 seconds. Accompanying transfusion management based on pointof-care testing, including thromboelastography and thromboelastometry in order to the dynamic process of blood coagulation was routinely performed. HIT developed in 5 patients in which Argatroban was continuously employed with an aPTT of 50-60 seconds. Perioperatively, erythrocyte and thrombocyte concentrates were administered at blood hemoglobin levels of $8 \mathrm{mg} / \mathrm{dL}$ and 80,000 thrombocytes/ $\mu \mathrm{L}$, respectively.

Transthoracic echocardiography was performed perioperatively in order to assess left and right ventricular size and function, position of ventricular septum, and inferior vena cava diameter for optimal fluid management.

All thoracic surgical procedures were performed in presence and guidance of a dedicated VAD coordinator of 
the implanting institution.

\section{Thoracic surgical management}

In all patients, video-assisted thoracoscopic surgery (VATS) was initially performed with a uni- or bi-portal approach. Severe pleuropulmonary adhesions were found in 12 patients which necessitated a conversion to thoracotomy. VATS allowed the exploration of the pleural cavity, removal of pleural empyema, disruption of septations and removal of pleural hematoma. Thoracoscopic or open decortication of the lung and parietal pleurectomy were performed with the aim of complete lung reexpansion and debridement of the thoracic cavity. The accompanying inflammatory peel was removed from the visceral and parietal pleura using blunt or sharp dissection with special caution to avoid lung injury or additional bleeding. In case of destroyed lung parenchyma due to carnified pneumonia or abscess formation, the affected area was resected using linear stapling devices. Superficial parenchymal bleeding or chest wall bleeding was managed using electrocautery or argon plasma cauterization. In all cases, intense pleural rinsing with a sodium hypochlorite antiseptic solution was performed in all primary and secondary procedures (Lavanox ${ }^{\circledR}$, SERAG WIESSNER GmbH, Naila, Germany). In the case where diffuse bleeding persisted, packing and temporary closure of the thoracic cavity was performed. Second look procedures were routinely performed in the operating room on a threeday schedule until no further signs of infection or bleeding were seen.

Two chest tubes were placed and were connected to a digital drainage system at a pressure of $-10 \mathrm{mmHg}$. Chest tubes were removed when an air leak was absent, drainage effusion was less than $150 \mathrm{~mL}$ within a 24-hour period and no positive microbiological exams on swabs taken from the chest drain site were observed.

\section{Statistical analysis}

Data were analyzed using the statistical program GraphPad Prism 7 (GraphPad Software, USA). Categorial variables were described as absolute and relative frequencies. Continuous variables were described using means, standard deviations, and ranges.

\section{Results}

Thirty-three patients ( 2 females), were identified and included in the analysis. The mean age was 65 years (49-78 years). VAD types implanted were HeartMate 3 (Abbott, Minneapolis, 5 patients) and HeartWare HVAD (Medtronic, Minneapolis, 26 patients), both for left ventricular support (LVAD). Indication for LVAD implantation were ischemic cardiomyopathy (24 patients) and dilative cardiomyopathy ( 7 patients). $\mathrm{N}=2$ patients with acute right ventricular failure required right ventricular assist device (RVAD) implantation. As surgical access, median sternotomy was performed in 23 patients and a left sided thoracotomy in 10 patients. Acquired von Willebrand disease after VAD implantation was observed in 5 patients.

VATS exploration of the thoracic cavity was performed in all 33 patients. Left-sided VATS was performed in 17 and right-sided VATS in 10 patients. $\mathrm{N}=6$ patients underwent bilateral surgical intervention. In 12 patients, conversion to thoracotomy was necessary due to the intraoperative presence of severe adhesions and/or diffuse bleeding. Decortication and parietal pleurectomy in addition to hematoma and empyema removal was performed in all patients. Two patients developed perforated lung abscess formations and secondary empyema. They were localized in the middle and right lower lobe, respectively. Complete resection of the middle lobe $(\mathrm{n}=1)$ and a basal segmentectomy (Segment 10) of the right lower lobe $(n=1)$ were performed. One patient showed therapy-refractory destroyed lung syndrome and sepsis due to carnified pneumonia which required a right lower lobe resection.

In 10 patients with diffuse bleeding, packing of the thoracic cavity with temporary thoracic closure was necessary. Re-exploration with redo-packing was performed in 8 patients and twice in 2 patients. Definitive chest closure was performed after a mean of 3 days (3-7 days). In none of the patients was surgical revision after definitive thoracic closure necessary. Figure 1 depicts preoperative radiological findings in a patient who received LVAD due to dilative cardiomyopathy and who developed postoperative massive left-sided hemothorax. VATS alone was not sufficient due to diffuse bleeding intraoperatively. Consequently, conversion to thoracotomy was performed and pleural packing was necessary to control the bleeding. After three days, depacking was successful with no further need for surgical revision.

Two patients died postoperatively due to septic shock with multiorgan failure. The 90-day mortality was $6 \%$ ( 2 patients). The mean ICU stay was 6 days (2-24 days). Chest tubes were removed 10 days (6-18 days) after the initial surgery. 

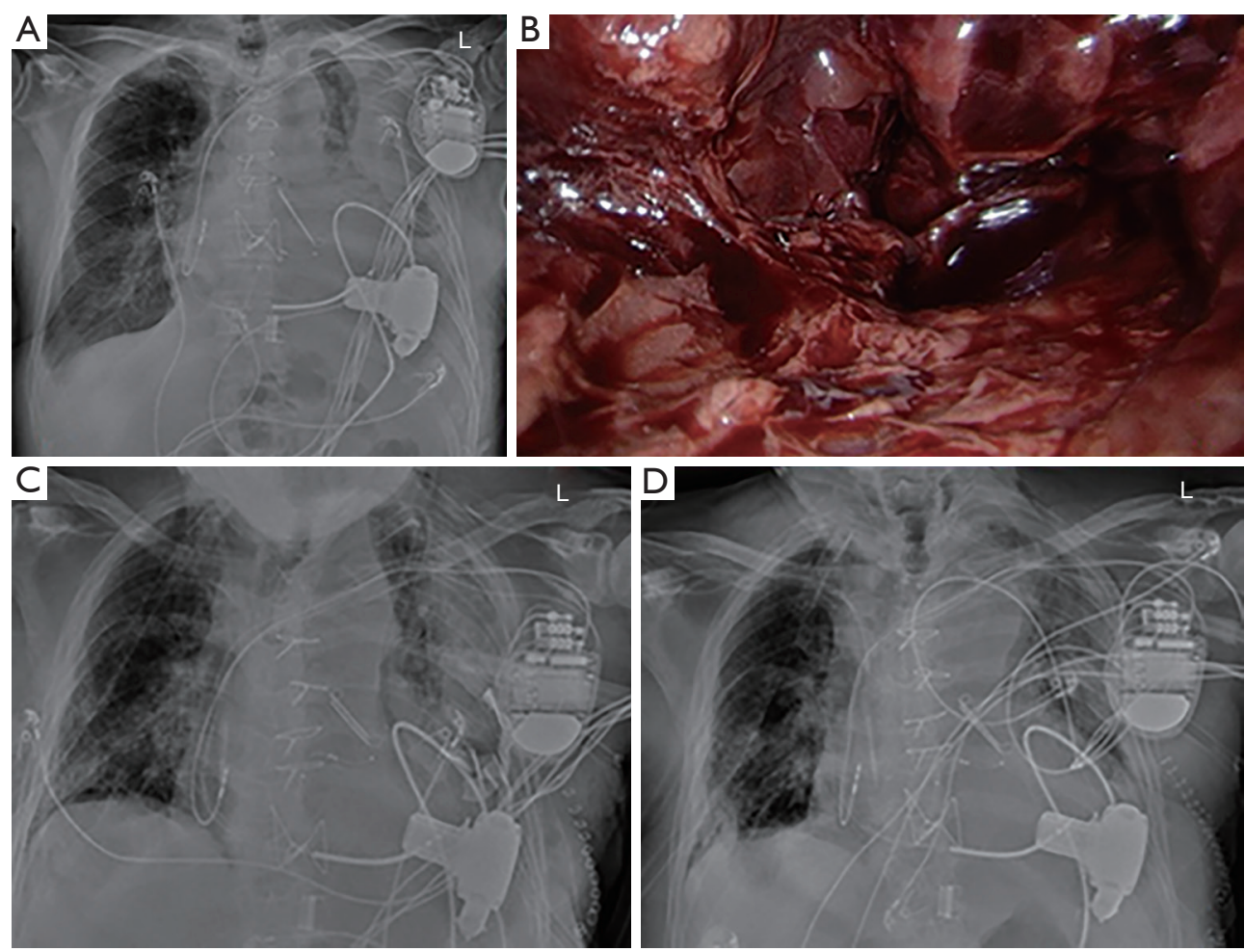

Figure 1 Perioperative findings in a patient who received LVAD due to dilative cardiomyopathy and who developed postoperative massive left-sided hemothorax. (A) Preoperative chest X-ray shows massive hemothorax and almost complete atelectasis of the left lung. (B) Intraoperative thoracoscopic view with evidence for partly solid and partly liquid infected hematoma with accompanying pleura empyema (fibrinopurulent phase). (C) Postoperative chest X-ray after conversion to thoracotomy and packing of the thoracic cavity due to intraoperative diffuse bleeding. (D) Chest X-ray after Depacking and definitive chest closure shows an optimal inflation of the left lung without evidence for hemothorax or pleural effusion.

$\mathrm{N}=4$ patients had a tracheostomy performed preoperatively due to respiratory failure. In these patients, chronic obstructive pulmonary disease (COPD) and impaired lung function was diagnosed prior to VAD surgery. These patients however, were successfully weaned of the respirator and decannulated after transfer to a weaning center.

One patient was readmitted to our department with acute secondary bleeding after discharge with no signs of bleeding after initial VATS. This time, thoracotomy and packing of the thoracic cavity due to diffuse bleeding was necessary. After 3 days, depacking and closure of the thoracic cavity was successfully performed.

One patient developed deep wound infection after thoracotomy which required vacuum assisted closuretherapy. This was managed with vacuum assisted closuretherapy (VAC therapy). Secondary wound closure was achieved 15 days after initiating the VAC therapy.

Histopathological examination confirmed the presence of pleura empyema stage 1 (exudative) in 5 patients, stage 2 (fibrinopurulent) in 19 patients, and stage 3 (chronic organizational) in 9 patients.

Microbiological examinations of pleural effusion, empyema, pleural hematoma or chest tube swaps revealed the presence of staphylococcus aureus in 6 patients, streptococcus pneumonia in 5 patients, klebsiella pneumonia in one patient and pseudomonas aeruginosa in one patient. Furthermore, candida albicans was present in one patient and aspergillus fumigatus in 3 patients. For the remaining 16 patients, pleural fluid was aseptic.

\section{Discussion}

Here we present our experience and concept of the thoracic surgical management of pleural infection in patients after VAD implantation for end-stage heart failure. After failing conservative treatment options, patients underwent 
decortication and pleurectomy as well as evacuation of a hemothorax and empyema using a thoracoscopic or open approach. Intraoperative trend to diffuse bleeding due to massive intrapleural inflammation and strict systemic anticoagulation therapy, required packing of the thorax for bleeding control in some patients.

It has been reported that patients with heart failure are already exposed to oxidative stress prior to VAD implantation. After VAD implantation, a continuous nonphysiological mechanism affecting the circulating blood is observed due to the shear stresses resulting from direct contact of blood with the artificial surface of VAD systems. This might lead to increased oxidative stress and leukocyte activation resulting in a significant inflammatory response, which possibly contributes to an escalation of pre-septic conditions especially after device implantation $(7,8)$.

The pathogenesis of pleural empyema, however, is not fully clarified. It is assumed that the development of empyema may be due to germ migration caused by adjacent lung infections or pneumonia into the pleural space (6). Other possible reasons are thoracic interventions or prolonged chest tube therapy (7).

The initial exudative stage of pleural empyema begins with increased interstitial fluid leakage due to inflammation with increased capillary permeability. Progression may lead to the development of multiple cavities resulting in pleural infection (fibrinopurulent, stage 2). At a later stage (chronic organizational phase, stage 3), proliferation of fibroblasts and thickening of the pleura develops as an expression of chronic inflammation $(6,9)$. Often, the surgical strategy is determined by the present stage of empyema. In early stage empyema, the British Thoracic Society (BTS) guidelines recommend chest tube drainage, antibiotic therapy and fibrinolytic drug application, however, failure of these in the presence of persistent pleural collection frequently requires surgical intervention (10). No consensus currently exists regarding the role of surgery as a first line approach. However, several studies suggested that VATS offers superior outcomes when compared with thoracotomy or chest tube insertion alone (11-13). For the early management of empyema and hemothorax after unsuccessful chest tube therapy, VATS was reported to be very successful. Conversion to thoracotomy becomes very often necessary when chronic empyema is present (13). In our study, conversion to thoracotomy was necessary, when stage 3 empyema and/or severe adhesions were present. Furthermore, the trend to diffuse bleeding in these patients requiring therapeutic systemic anticoagulation, led to the decision to convert to open surgery in certain cases.

In patients that require mechanical ventilatory support, ventilation associated pneumonia may play a role in the development of empyema and lung abscess formation. In our study cohort, 4 patients were presented with tracheostomy performed after VAD implantation due to postoperative respiratory failure and were mechanically ventilated. Those patients could be then successfully weaned off the respirator and decannulated after thoracic surgical intervention.

Infection, bleeding, pump thrombosis and stroke are the most serious complications of permanent VAD circulatory support $(3,4)$. According to recent INTERMACS report, both ischemic and hemorrhagic strokes pose the major cause of death between 6 months and 4 years after VAD implantation. Bleeding complications are mainly gastrointestinal and represent the major cause of both early and late morbidity in VAD patients (3). Reports concerning the postoperative management of hemothorax after VAD implantation are rare. No larger patient cohort has been reported to date.

When bacterial infection occurs, increased vascular permeability and local release of inflammatory mediators may result in pleural cavity reaction and consequently fluid accumulation with a high number of inflammatory cells (14). In context of VAD implantation and the immunomodulatory nature of end-stage heart failure, infection and inflammation of the pleural cavity may represent an increased risk for primary or secondary device infection. Therefore, any delay of surgical intervention in case of suspected infection of the pleural cavity may promote poor outcome. In particular, this may also lead to blood stream infections which is in the majority of cases difficult to treat due to the presence of implanted artificial devices and is associated with increased mortality. Blood stream infections may be also associated with cerebrovascular complications (15). Furthermore, VAD patients with blood stream infection are more likely to develop haemorrhagic and ischemic events than patients without infection (16). In our patient cohort, two patients died due to sepsis with multiorgan failure. Nevertheless, in the remaining patients, reduced mortality rate was observed despite the acuity of illness in these patients. Therefore, it can be assumed that an early thoracic surgical intervention may lead to decreased risk of blood stream infections.

Management of pleural infections and hemothorax in VAD patients remains a challenge. The need for surgical revision due to postoperative bleeding during LVAD support is addressed to influence postoperative mortality 
and morbidity. Recently, Angleitner et al. reported that most early and late postoperative revisions are mainly due to intraoperative diffuse bleeding. The most common causes were bleeding from the LVAD outflow graft anastomosis and thoracic wall (17). The concomitant presence of pleuritis and inflammation resulting from parapneumonic effusions and pleural empyema leads to hyperaemia and significant vascular proliferation of the pleura. This may influence hemorrhage of the pleural cavity making surgical intervention and perioperative coagulation management challenging (18).

Meticulous surgical exploration of the thoracic cavity, even to identify minuscule bleeding from the thoracic wall seems to be important. For this purpose, minimally invasive VATS is feasible and less traumatic. In the case of ongoing diffuse bleeding, the surgical field can be packed with sterile gauze until coagulation management takes effect (17). In this study, chest tubes were placed in all patients prior to the decision for surgical intervention. However, chest tube therapy of a large hemothorax or pleural empyema is difficult and was unsuccessful in those patients. Due to the pronounced pleural inflammation and despite optimization of systemic coagulation, persistent diffuse bleeding intraoperatively led to the decision of packing the thoracic cavity. After resolution of the infection, re-exploration of the thoracic cavity was performed. If no bleeding was observed, the chest was closed. Otherwise, redo-packing was performed.

VAD implantation has become an accepted and routinely applied treatment option for patients with end-stage heart failure. However, it remains a challenge, even in specialized high-volume centers. This is mostly due to the fact that the underlying disease and consequent chronic cardiac failure leads to significantly reduced physical patient conditions, which itself affects outcome after surgery. It also often leads to impairment of other organ functions such as the liver, kidney, brain and the immune system including regulation of inflammatory response. In addition to major surgery in these compromised patients and the implantation of a device with artificial blood-contacting surfaces, therapeutic anticoagulation is required, all of which contribute to an increased risk of thoracic bleeding and infection. Whereas VAD implantation represents and requires cardiac surgical expertise, the management of the challenging postoperative complications, requires the expertise of thoracic surgery. In order to generate the best possible outcome after VAD implantation, we have developed this interdisciplinary strategy for the management of extracardial but intrathoracic complications after VAD implantation, which to our knowledge is the first report on such an interdisciplinary approach.

\section{Acknowledgments}

The authors thank Dr. Ali Hasan (MidMichigan Medical Center-Alpena) for language editing throughout the manuscript and for statistical review of the data. The results of this study have in parts been presented at the Joint Annual Congress of the German, Austrian and Swiss Society of Thoracic Surgery (29.09-01.10 2019) in Salzburg, Austria.

Funding: None.

\section{Footnote}

Reporting Checklist: The authors have completed the STROBE reporting checklist. Available at https://dx.doi. org/10.21037/jtd-20-2886

Data Sharing Statement: Available at https://dx.doi. org/10.21037/jtd-20-2886

Conflicts of Interest: All authors have completed the ICMJE uniform disclosure form (available at https://dx.doi. org/10.21037/jtd-20-2886). The authors have no conflicts of interest to declare.

Ethical Statement: The authors are accountable for all aspects of the work in ensuring that questions related to the accuracy or integrity of any part of the work are appropriately investigated and resolved. The study was conducted in accordance with the Declaration of Helsinki (as revised in 2013). The study is a retrospective analysis of routine clinical data with no information regarding personal data such as name, address, or pictures. According to our local ethic committee, no ethical approval was required prior to this study. Informed consent for performing the surgical procedures was taken from all patients prior to surgery.

Open Access Statement: This is an Open Access article distributed in accordance with the Creative Commons Attribution-NonCommercial-NoDerivs 4.0 International License (CC BY-NC-ND 4.0), which permits the noncommercial replication and distribution of the article with the strict proviso that no changes or edits are made and the 
original work is properly cited (including links to both the formal publication through the relevant DOI and the license). See: https://creativecommons.org/licenses/by-nc-nd/4.0/.

\section{References}

1. Hunt SA, Abraham WT, Chin MH, et al. ACC/AHA 2005 Guideline Update for the Diagnosis and Management of Chronic Heart Failure in the Adult: a report of the American College of Cardiology/American Heart Association Task Force on Practice Guidelines (Writing Committee to Update the 2001 Guidelines for the Evaluation and Management of Heart Failure): developed in collaboration with the American College of Chest Physicians and the International Society for Heart and Lung Transplantation: endorsed by the Heart Rhythm Society. Circulation 2005;112:e154-235.

2. Cai AW, Islam S, Hankins SR, et al. Mechanical Circulatory Support in the Treatment of Advanced Heart Failure. Am J Transplant 2017;17:3020-32.

3. Kirklin JK, Pagani FD, Kormos RL, et al. Eighth annual INTERMACS report: Special focus on framing the impact of adverse events. J Heart Lung Transplant 2017;36:1080-6.

4. Schramm R, Morshuis M, Schoenbrodt M, et al. Current perspectives on mechanical circulatory support. Eur J Cardiothorac Surg 2019;55:i31-7.

5. Simon D, Fischer S, Grossman A, et al. Left ventricular assist device-related infection: treatment and outcome. Clin Infect Dis 2005;40:1108-15.

6. Light RW. Parapneumonic effusions and empyema. Proc Am Thorac Soc 2006;3:75-80.

7. Mondal NK, Sorensen E, Hiivala N, et al. Oxidative stress, DNA damage and repair in heart failure patients after implantation of continuous flow left ventricular assist devices. Int J Med Sci 2013;10:883-93.

8. Mondal NK, Sorensen EN, Pham SM, et al. Systemic

Cite this article as: Akil A, Rehers S, Köhler C, Richter L, Semik M, Ziegeler S, Fischer S. Multidisciplinary management of pleural infection after ventricular assist device implantation. J Thorac Dis 2021;13(8):4661-4667. doi: 10.21037/jtd-20-2886
Inflammatory Response Syndrome in End-Stage Heart Failure Patients Following Continuous-Flow Left Ventricular Assist Device Implantation: Differences in Plasma Redox Status and Leukocyte Activation. Artif Organs 2016;40:434-43.

9. Sahn SA. Diagnosis and management of parapneumonic effusions and empyema. Clin Infect Dis 2007;45:1480-6.

10. Davies HE, Davies RJ, Davies CW, et al. Management of pleural infection in adults: British Thoracic Society Pleural Disease Guideline 2010. Thorax 2010;65 Suppl 2:ii41-53.

11. Chan DT, Sihoe AD, Chan S, et al. Surgical treatment for empyema thoracis: is video-assisted thoracic surgery "better" than thoracotomy? Ann Thorac Surg 2007;84:225-31.

12. Tong BC, Hanna J, Toloza EM, et al. Outcomes of videoassisted thoracoscopic decortication. Ann Thorac Surg 2010;89:220-5.

13. Landreneau RJ, Keenan RJ, Hazelrigg SR, et al. Thoracoscopy for empyema and hemothorax. Chest 1996;109:18-24.

14. Kroegel C, Antony VB. Immunobiology of pleural inflammation: potential implications for pathogenesis, diagnosis and therapy. Eur Respir J 1997;10:2411-8.

15. Kanjanahattakij N, Horn B, Abdulhadi B, et al. Blood stream infection is associated with cerebrovascular accident in patients with left ventricular assist device: a systematic review and meta-analysis. J Artif Organs 2018;21:271-7.

16. Toda K, Yonemoto Y, Fujita T, et al. Risk analysis of bloodstream infection during long-term left ventricular assist device support. Ann Thorac Surg 2012;94:1387-93.

17. Angleitner P, Simon P, Kaider A, et al. Impact of Bleeding Revision on Outcomes After Left Ventricular Assist Device Implantation. Ann Thorac Surg 2019;108:517-23.

18. Karpathiou G, Hathroubi S, Patoir A, et al. Non-specific pleuritis: pathological patterns in benign pleuritis. Pathology 2019;51:405-11. 